\title{
PYMES COLOMBIANAS Y LOS RETOS DE LA INDUSTRIA 4.0
}

\section{COLOMBIAN SMES AND INDUSTRY 4.0 CHALLENGES}

\author{
Greys Yuranis Chalarca Arboledal \\ Sandra Bibiana Hurtado Perdomo ${ }^{2}$ \\ Edward Fabián Escovar Álvarez ${ }^{3}$
}

Cómo citar este artículo: Chalarca Arboleda, G.Y., Hurtado Perdomo, S. B. \& Escovar Álvarez, E. F. (2020).

Pymes colombianas y los retos de la industria 4.0. Revista Innova ITFIP, 7(1),10-17.

Recibido: Junio de 2020 Aprobado: Diciembre de 2020

\section{Resumen}

Este artículo presenta elementos generales de un proyecto de investigación en curso el cual está orientado a proponer un modelo o metodología que permita lograr un acercamiento y aprovechamiento de las herramientas que trae consigo la nueva era digital por parte de un grupo focal de Pymes Colombianas, con el fin de fortalecer sus procesos productivos y facilitar la adaptación y transformación de estas a los nuevos retos de la creciente industria 4.0.

Palabras Clave: Pymes, Industria 4.0, desarrollo empresarial, transformación digital.

\begin{abstract}
This article presents general elements of an ongoing research project which is aimed at proposing a model or methodology that allows to achieve an approach and use of the tools that brings with it the new digital age by a focus group of Colombian SMEs, in order to strengthen their production processes and facilitate the adaptation and transformation of these to the new challenges of growing industry 4.0.

Keywords: SMEs, Industry 4.0, business development, digital transformation.

\section{Introducción}

Las empresas a lo largo de la historia han estado ligadas a constantes avances en sus procesos productivos, desde la primera revolución industrial el concepto de administración y de empresa empezó a adquirir otro significado, y a medida que cada revolución se va desarrollando aparecen nuevas herramientas y tecnologías que pretenden mejorar y aumentar la producción de las organizaciones. Cada revolución industrial trajo consigo grandes oportunidades, pero también grandes retos que ponen a prueba la capacidad de las empresas para adaptarse a dichos cambios,
\end{abstract}

\footnotetext{
${ }^{1}$ Especialista en Gestión de proyectos. Administradora de Empresas de la Fundación Universitaria San Martín. Investigadora en Grupo de Investigación CIECAD - Universitaria Virtual Internacional. Correo: gychalarcaa@uvirtual.edu.co (https://orcid.org/0000-0001-7200-6557)

2 Especialista en Gerencia Educativa. Administradora de Empresas de la Universidad Nacional de Colombia Sede Manizales. Investigadora en Grupo de Investigación CIECAD - Universitaria Virtual Internacional. Correo: sbhurtadop@uvirtual.edu.co (https://orcid.org/0000-0002-5540-9917)

${ }^{3}$ Maestrante en Educación. Especialista en Alta Gerencia. Contador Público y Administrador de Empresas de la Universidad Nacional de Colombia. Director Grupo de Investigación CIECAD - Universitaria Virtual Internacional. Correo: efescovara@uvirtual.edu.co (https://orcid.org/0000-0001-9031-2590)
} 
las empresas con estructuras organizacionales muy rígidas o mal estructuradas con procesos inflexibles están tendiendo a desaparecer.

Para Colombia, el comportamiento de los mercados y de las organizaciones no es ajeno a esta realidad. Las empresas en todos los sectores y de todos los tamaños se enfrentan día a día con consumidores más exigentes que buscan diversificar su experiencia al momento de consumir un producto o servicio. Aspectos como la facilidad, flexibilidad, agilidad y accesibilidad entre otros, se pueden convertir en una ventaja competitiva para las organizaciones

En Colombia se cuenta con una estructura empresarial formada en su mayoría de empresas en pequeña escala, dichas empresas pequeñas y medianas son denominadas Pymes (Murillo \& Restrepo, 2016). El sector de las pymes representa un segmento bastante grande en la economía nacional, según el DANE estas conforman un 90\% del sector productivo del país y están generando el 80\% del empleo con una tasa representativa del PIB de un 35\% (MinTrabajo, 2019); Las pequeñas y medianas empresa no solo generan un tercio del PIB, también son el pináculo de la generación de empleo del país, estas permiten el mejoramiento de la calidad de vida de los colombianos siendo vitales para sostener el crecimiento económico del país a largo plazo y con un gran potencial para generar desarrollo y expiación económica (Nataliya Mylenko, 2012), pero estas también enfrentan una tasa bastante alta de fracasos .

La tasa de fracaso de las Pymes en Colombia está en un 16\% anual (RomeroEspinosa, Molinab, \& AnalíVera-Colinac, 2015), y el número de empresas que desaparece aumenta a un 50\% al llegar a los 5 años, teniendo en cuenta que las Pymes ofrecen en oportunidad de empleo y desarrollo económico es de vital importancia entender los retos que enfrentan actualmente y brindar herramientas que permitan ampliar sus mercados, generando de esta forma sostenibilidad y mejore su competitividad permitiendo que estas puedan aumentar su capacidad de éxito a través del tiempo y los nuevos entornos que ofrecen la industria a nivel nacional e internacional, todo esto, teniendo en cuenta a (Barrios Zarta, J. 2019; Batista Castillo, A., Guacari Villalba, W. D. 2018).

Muchos de los problemas que enfrenta este sector, radica en su capacidad de adaptación a las demandas de la industria y sus mercados, sus procesos de gestión que en muchos casos tienen grandes fortalezas en las áreas operativas de su actividad económica también manifiestan muchas falencias en demás áreas como contabilidad, finanzas, marketing gestión de personal (Vera-Colina, Melgarejo-Molina, \& Mora-Riapira, 2014) ; esto permite entender sus debilidades al enfrentase a una sociedad y mercados que está en constante cambio.

En un entorno comercial cambiante, la cuarta revolución industrial da pasos agigantados en lo que lo que será una nueva era de negocios y oportunidades para todas las empresas; cada momento de la historia reciente está marcado por una revolución industrial, esto permitió dar saltos enormes en los procesos productivos de sus épocas y la implementación de tecnologías, generando grandes mejoras en tiempos de fabricación, innovaciones en metodologías de producción; impulsando de esta forma el comercio a nivel mundial. 
REVISTA INNOVA ITFIP, 7 (1). 10-17. DIC. 2020

En la nueva era de la digitalización y medios virtuales todas las organizaciones del mundo definen estrategias que les permitan alcanzar un crecimiento inteligente en lo que hoy se conoce como la Industria 4.0, dichas estrategias buscan adaptar los nuevos desarrollos en tecnologías, sistemas de producción, transformación digital y desarrollo de software que permitan la automatización de los sistemas de interacción entre las organizaciones y el consumidor. Tomando en cuenta a Peña, C., Velázquez, L., \& García, 2017; Varón, O., Gutiérrez, D. \& Rodríguez, W. 2018); Castellanos, P.\& Escott, M. 2020).

Gracias a muchas de las herramientas de la industria 4.0, son infinitas las formas en que una empresa puede consolidarse en el mercado y aumentar sus oportunidades de éxito. Actualmente en Colombia, se cuenta con espacios como "Colombia 4.0", un evento dirigido a las organizaciones y emprendedores para dar a conocer sus ideas y compartir información sobre las oportunidades en esta nueva era de la transformación digital, gracias a la alianza entre el Ministerio de Tecnologías de la Información y las Comunicaciones; el Ministerio de Comercio, Industria y Turismo, e iNNpulsa para unir a Colombia 4.0. y Héroes Fest. (MinTIC, 2019), cada vez más es evidente la integración de nuevas política pública y sectores de manufactura, agro-industria y servicios, y existe un amplio consenso en cuanto a la importancia de la Industria 4.0 para el futuro de la economía del país y oportunidades para las Pymes.

Teniendo en cuenta lo anterior, se pretende desarrollar una metodología para el aprovechamiento del potencial de transformación digital en un grupo focal de Pymes colombianas, teniendo como referencia la innovación como herramienta para el desarrollo sostenible y estratégico de las mismas.

\section{Metodología}

De manera que se pueda cumplir el objetivo planteado en esta investigación se plantea en la primera fase una exploración y análisis cualitativo de modelos actuales que permitan a las Pymes determinar su estado actual en procesos relacionados con la transformación digital. Dicho análisis se centra en la revisión teórica de artículos y textos que relacionan los avances de la industria 4.0, algunos modelos de transformación digital y los retos que pueden representar para las Pymes Colombianas la implementación de estas herramientas en sus procesos organizacionales.

\section{Marco Teórico}

Las pequeñas y medianas empresas luchan día a día por ser más competitivas en el mercado, en una investigación sobre el fracaso empresarial de las pequeñas y medianas empresas (PYMES) en Colombia (RomeroEspinosa, Molinab, \& AnalíVera-Colinac, 2015) para las variables financieras que explican la situación de fracaso de las (pymes) en Colombia. Nos muestran que son muchos los factores externos que afectan la capacidad de éxito y fracaso de una empresa, pero 
en los más relevantes esta, disponibilidad de mecanismos de crédito, los trámites administrativos, las relaciones con el sector gubernamental, entidades financieras y proveedores, además de malos proceso administrativos y organizacionales selección de personal problemas con sus modelos de marketing.

(Mora \& Bravo, 2009) Remarca la importancia de que las Pymes cuenten con las herramientas adecuadas para poder generar una mejora en la taza de éxito ya que en una situación de constante fracaso puede generar una inestabilidad social en el país debido al gran número de empleos que generan las Pymes, de esta forma propusieron aplicación de una de una técnica de escalado Multidimensional a la estructura de factores económicos y financieros de dos grupos de empresas ha permitido identificar cuáles son los rasgos subyacentes bajo las situaciones asociadas al fracaso y desaparición de empresas, así como a la continuidad de la actividad, en un conjunto de empresas manufactureras de Bogotá en el período 1993-2005. Su modelo nos permite identificar que las Pymes cuentan con una gran capacidad de adaptación y que unos delos factores que más influyes es la falta de conocimiento para aplicar dichas herramientas ya sea financieras, organizacionales o de marketing.

La Asociación Colombiana de las Micro, Pequeñas y Medianas Empresas (ACOPI) resalta la importancia de Combinar las técnicas avanzadas de producción y operaciones con tecnologías inteligentes que se integrarán en las organizaciones, las personas y los activos. Denotando que permitirá una mayor individualización de la oferta y mayor flexibilidad de los procesos de producción y, para ello, es preciso que toda la información derivada del proceso de fabricación esté disponible en tiempo real en todos los departamentos y áreas de la organización. (ACOPI, 2020) Analizar los desafíos de la cuarta revolución industrial y concertar acciones para fortalecer la formación académica, entidades como el SENA muestran la importancia del desarrollo de la agenda académica incluye otros temas como: robótica colaborativa, perspectiva de tecnologías, industria automotriz, internet de las cosas y realidad mixta. Además, se realizarán talleres en relación a los retos de la formación profesional y las mesas sectoriales frente a la cuarta revolución industrial. (Mogollón \& Martin, 2018)

Con el fin de generar una estrategia o modelo de transformación en las organización hacia la Industria 4.0 (Prieto \& Bedoya, 2019), presenta una investigación enfocada a la recolección de información cualitativa que permita identificar modelos de aplicación al sector productivo con el fin de mejorar sus operaciones, Presenta una tabla de las empresa, su actividad y como se ha destacado en la innovación según su sector.

En los modelos productivos encontramos un proyecto de aplicación basado en el sistema lean manufacturing e industria 4.0 para mejorar el sistema de producción automatizado y semiautomatizado en la empresa cilindros Company S.A.S. en la ciudad de Neiva (Vasquez, Farfán, Charry, \& Gutierrez, 2019), donde se interviene una línea de producción de cilindros, en él participan 4 máquinas de automatizado que trabajan con un sistema de producción modular pero presentaba problemas con su funcionamiento debido a falencia del personal con relación a 
el lenguaje de programación de las máquinas, a su vez, se empleó mucho tiempo en el levantamiento de las señales de los sensores y los actuadores de cada estación, limitando el tiempo para la puesta en marcha de todo la maquina en conjunto; bloqueo del regulador de presión siendo este un daño leve en la máquina evitando que el proceso fluyera normalmente.

\section{Resultados y Discusión}

Colombia no es el único país a nivel latinoamericano que apunta a la nueva industria 4.0 como herramienta de apoyo para generar desarrollo económico en las Pymes, (Motta, Morero, \& Ascúa, 2019) presenta parte de un estudio sobre industria 4.0 y su impacto sobre las pymes industriales en ciertas regiones de América Latina liderado por la Universidad Nacional de Rafaela en Argentina. El objetivo de la investigación fue indagar explorar sobre las motivaciones, beneficios y obstáculos a la introducción de tecnologías 4.0 en pymes industriales de la Argentina, como un punto de partida para el diseño de políticas.

La investigación arrojó diversos "gradientes" de adopción de las tecnologías 4.0, que responden en parte a las específicas necesidades estratégicas de competencia de cada empresa (en la mayoría de los casos estudiados la resolución de algún problema), y en otra parte a que determinados obstáculos limitan una mayor profundización de la digitalización. La adopción de tecnologías 4.0 no es necesariamente una alternativa binaria; por el contrario, en la totalidad de los casos analizados en ese estudio se verifica una adopción parcial de estas nuevas tecnologías. (Motta, Morero, \& Ascúa, 2019)

Colombia en materia tecnológica se encuentra en la posición número 47 dentro de un total de 118 en el mundo, y a nivel latinoamericano ocupa la posición número 7 (ACOPI, 2020); si bien aún queda mucho trabajo por hacer, uno de los factores más importantes es poder determinar metodologías que permitan a las Pymes y organizaciones del país conocer estas tecnologías y herramientas para generar una transición a la nueva industria 4.0 de la manera más eficiente posible.

\section{Metodologías relacionadas con la transformación digital en las organizaciones}

Durante la exploración teórica fue posible identificar algunos modelos existentes y herramientas que se han demostrado tener gran acogida en el contexto organizacional gracias a los resultados evidenciados en su aplicación. A continuación se relacionan algunas herramientas de análisis, como por ejemplo, el modelo Canvas, la cual tiene como fin determinar el valorar de una idea si esta es eficaz y eficiente, definiendo todas características más importantes por medio de un diagrama de nueve módulos de contenido plasmados en un lienzo de manera estructurada, visual y a modo de resumen. (Ferreira-Herrera, 2015). 
También se encuentra la metodología de transformación digital (3O3P), la cual se enfoca en el tarto con los clientes, marketing y gestión de la calidad como premisas que permitirán hacer una revisión del estado actual de la organización y establecer las estrategias más adecuadas para llegar a la Industria 4.0. (Israel García, 2020)

Por otro lado se referencia la metodología de transformación digital AMETIC, la cual plantea que la transformación digital no solo es la implementación de tecnologías en la empresa, sino que representa también un cambio cultural en las organizaciones y sus proceso es la oportunidad de reinventarse para adaptarse a los nuevos retos que trate la cuarta revolución industrial y aprovechar al máximo las nuevas herramientas con las que se cuentan. (AMETIC, 2017)

Por último pero no menos importante se relaciona el modelo o metodología sugerido para el proceso de transformación digital por parte de La Red internacional de Universidades Virtuales, Red SUMMA, donde se plantean a través de una serie de pasos o recomendaciones la mejor manera de ayudar a las empresas y organizaciones en sus procesos de digitalización (Internacional, 2020).

\section{Conclusiones}

Durante esta primera fase exploratoria ha sido posible evidenciar un alto grado de interés de los diferentes actores por encontrar mejores maneras de adaptarse a la nueva realidad digital, desde los gobiernos hasta el sector productivo acompañados de la academia están en constante comunicación para generar espacios de transferencia de conocimiento y socialización de buenas prácticas en pro del fomento de la transformación digital y su impacto en el desarrollo social de las naciones. Lo anterior se convierte en una oportunidad de aportar y contribuir con estos esfuerzos a través de la investigación.

Si bien es cierto, se resalta el interés por parte de las organizaciones por transformar sus procesos y aprovechar las nuevas herramientas digitales para incrementar su competitividad en el mercado, es posible evidenciar que debido a la aceleración de la implementación de la llamada industria 4.0 algunas organizaciones en especial las Pymes aún carecen del conocimiento y la capacidad para asumir los retos que esta nueva realidad representa.

Por otra parte, de acuerdo con un reciente informe de la comisión económica para américa Latina y el Caribe CEPAL, el 88\% de las empresas formales en américa Latina son microempresas, las cuales debido a la reciente pandemia generada por el SARS-COV-2 la mayoría de ellas se verá afectada en sus ventas y tienen alta probabilidad de ser insolventes en un corto periodo de tiempo. "Aunque la crisis afecta a todas las empresas, el impacto será mucho mayor en el caso de las microempresas y las pymes, por su peso en la estructura empresarial de la región, en que se traducirá en grandes cierres de empresas y pérdidas de puestos de trabajo" (CEPAL,2020). Teniendo en cuenta lo anterior sumado a las dificultades de las Pymes para adaptarse a la 
REVISTA INNOVA ITFIP, 7 (1). 10-17. DIC. 2020

digitalidad que ha demostrado ser una aliada para enfrentar la pandemia, podríamos intuir que la mortalidad de las Pymes podría ser mayor de lo estimado si no se le acompaña en esta transición.

Existen diferentes metodologías que sugieren buenas prácticas y resaltan elementos a considerar si se quiere migrar a la digitalidad dentro de un contexto organizacional, no obstante, no se evidencia una metodología o modelo específico adaptado a las necesidades de las Pymes, los modelos muestran herramientas y áreas en las cuales centrarse durante el proceso, no obstante, resultan ser muy genéricas y en ocasiones desconectadas del entorno real de las Pymes, no existe un consenso ni una ruta clara hacia la particularidad de cada organización delegando casi que la responsabilidad en el tipo de liderazgo con que se cuente y los recursos disponibles.

Conforme a los anteriores hallazgos se ha considerado oportuno continuar con la siguiente fase de la investigación, la cual pretende a través de una caracterización de un grupo focal de Pymes, determinar el potencial de transformación digital de sus procesos teniendo en cuenta sus condiciones y el contexto en el cual desarrollan su actividad económica, con el fin de ofrecer desde la academia y en compañía del sector productivo acompañamiento y soluciones efectivas que contribuyan al aprovechamiento de dicho potencial.

\section{Bibliografía}

ACOPI. (2020). Brechas Tecnológicas 4.0. Obtenido de https://acopi.org.co/wpcontent/uploads/2020/05/INFOGRAFIA-BRECHAS-TECNOL\%C3\%93GICAS.pdf

AMETIC. (2017). TRANSFORMACIÓN DIGITAL: Visión y Propuesta de AMETIC. España: AMETIC.

Barrios Zarta, J. (2019). Impacto de la innovación en la competitividad de las empresas del sector agroindustrial, en el Espinal-Tolima. Revista Innova ITFIP, 4(1), 7-20. Recuperado a partir de http://www.revistainnovaitfip.com/index.php/innovajournal/article/view/43.

Batista Castillo, A., Guacari Villalba, W. D. (2018). Gestión empresarial y competitividad en las mipymes del centro comercial Nueva Colombia de la ciudad de Cartagena. Revista Innova ITFIP, 3(1), 76-82. Recuperado a http://revistainnovaitfip.com/index.php/innovajournal/article/view/41.

Castellanos, P.; Escott, M. Evolución de las Habilidades Laborales en la Industria 4.0 y su Impacto Financiero. $\quad$ ITFIP 2020, 6, 106-119. Available online: http://revistainnovaitfip.com/index.php/innovajournal/article/view/82

Ferreira-Herrera, D. C. (14 de Junio de 2015). El modelo Canvas en la formulación de proyectos. Obtenido de file://C:/Users/Usuario/Downloads/1252-Texto\%20del\%20art\%C3\%ADculo-3085-1-1020160426.pdf

Internacional, R. S. (19 de junio de 2020). Pasos y Metodología para la Transformación Digital. Obtenido de $\quad$ https://campusvirtual.iep.edu.es/recursos/recursos_premium/programahabilidades/pdf/transformacion digital/contenido1/lectura3.pdf

Israel García, R. (16 de Junio de 2020). METODOLOGÍA DE TRANSFORMACIÓN DIGITAL (3O3P). Recuperado el 20 de junio de 2020 
MinTIC (7 de Noviembre de 2019) El futuro digital es de todos: https://www.mintic.gov.co/portal/inicio/Sala-de-Prensa/Noticias/107096:Colombia-4-0-inspiro-amas-de-4-000-personas-en-Barranquilla

MinTrabajo. (2019). "MiPymes representan más de 90\% del sector productivo nacional y generan el $80 \%$ del empleo en Colombia". https://www.mintrabajo.gov.co/web/guest/prensa/comunicados/2019/septiembre/mipymesrepresentan-mas-de-90-del-sector-productivo-nacional-y-generan-el-80-del-empleo-en-colombiaministra-alicia-arango.

Mogollón, Y. D., \& Martin, G. (2018). Gremios, empresas y SENA hacen frente a las industrias 4.0. Obtenido de https://www.sena.edu.co/es-co/Noticias/Paginas/noticia.aspx?IdNoticia $=3737$

Mora, M. C., \& Bravo, M. I. (2009). CARACTERIZACIÓN DEL FRACASO EMPRESARIAL. Universidad de Salamanca.

Motta, J., Morero, H., \& Ascúa, R. (2019). Industria 4.0 en mipymes manofactura de la Argentins. Obtenido de https://repositorio.cepal.org/bitstream/handle/11362/45033/1/S1900952_es.pdf

Murillo, L. M., \& Restrepo, S. L. (2016). Confecamaras red camara de comercio. Obtenido de http://www.confecamaras.org.co/phocadownload/Cuadernos_de_analisis_economico/Cuaderno_d e_An\%D0\%B0lisis_Economico_N_11.pdf

Nataliya Mylenko, V. S. (10 de Octubre de 2012). Pacific Economic Review; Access to Finance by Small and Medium Enterprises: a Cross-Country Analysis with A New Data Set. Obtenido de https://onlinelibrary.wiley.com/doi/abs/10.1111/j.1468-0106.2012.00596.x

Peña, C., Velázquez, L., \& Garcia, R. (2017). TECNOLOGÍAS DE INFORMACIÓN EN EL APRENDIZAJE EN LA FACULTAD DE CONTADURÍA Y ADMINISTRACIÓN - UAQ, MÉXICO. Revista Innova ITFIP, 1(1), 47-57. Recuperado a partir de $\mathrm{http}: / /$ revistainnovaitfip.com/index.php/innovajournal/article/view/12

Prieto, Y. E., \& Bedoya, E. Y. (2019). TRANSFORMACIÓN DEL MODELO 4.0 EN LOS SECTORES PRODUCTIVOS.

Obtenido

de https://repository.ucc.edu.co/bitstream/20.500.12494/16016/1/2019_transformaci\%C3\%B3n_mod elo_sectores.pdf

Romero Espinosa, F., Molinab, Z. A., \& AnalíVera-Colinac, M. (2015). Fracaso empresarial de las pequeñas y medianas empresas (pymes) en Colombia. Suma de Negocios.

Varón Giraldo, O., Gutiérrez Delgado, D. M., \& Rodríguez López, W. D. (2018). Procesos contables aplicados por los comerciantes al por menor del municipio de icononzo -tolima para identificar necesidades de capacitación en el área.Revista Innova ITFIP,3(1), 27-34. Recuperado a partir de http://revistainnovaitfip.com/index.php/innovajournal/article/view/36

Vasquez, A. D., Farfán, E. F., Charry, A. M., \& Gutierrez, O. A. (2019). IMPLEMENTACIÓN DE LEAN MANUFACTURING E INDUSTRIA 4.0 PARA. Obtenido de https://repository.ucc.edu.co/bitstream/20.500.12494/14439/1/2019_Lean_manufacturing_Cilindr os_company_S.A.S..pdf

Vera-Colina, M. A., Melgarejo-Molina, Z. A., \& Mora-Riapira, E. H. (2014). Accesos al finaciemiento en Pymes colombianas: una mirada desde sus indicadores financieros. INNOVAR. 